\title{
Source Image Based New 3D Rotational Angiography for Differential Diagnosis between the Infundibulum and an Internal Carotid Artery Aneurysm : Pilot Study
}

\author{
Hyeongyu Jang, ${ }^{1, *}$ Woo Sang Jung, ${ }^{2,3, *}$ Seong Uk Myoung, ${ }^{4}$ Jung-Jae Kim ${ }^{5}$ Chang Ki Jang, ${ }^{6}$ Kwang-Chun Cho ${ }^{1}$ \\ Department of Neurosurgery, International St. Mary's Hospital, College of Medicine, Catholic Kwandong University, Incheon, Korea \\ Department of Radiology, ${ }^{2}$ Ajou University Hospital, College of Medicine, Ajou University, Suwon, Korea \\ Department of Radiology, ${ }^{3}$ College of Medicine, Kangwon National University, Chuncheon, Korea \\ Department of Radiology, ${ }^{4}$ Bundang Jesaeng General Hospital, Sungnam, Korea \\ Department of Neurosurgery, ${ }^{5}$ Ewha Womans University Seoul Hospital, College of Medicine, Ewha Womans University, Seoul, Korea \\ Department of Neurosurgery, ${ }^{6}$ Yongin Severance Hospital, College of Medicine, Yonsei University, Yongin, Korea
}

Objective : Distinguishing between an infundibulum and a true aneurysm is clinically important. This study aimed to evaluate whether using source image based new three-dimensional rotational angiography (S-n3DRA) can increase the rate of aneurysm detection and improve distinction between a true aneurysm and an infundibulum.

Methods : Twenty-two consecutive patients with 23 lesions, were evaluated by time-of-flight (TOF) magnetic resonance angiography (MRA), S-n3DRA, and digital subtraction angiography (DSA). The data were retrospectively and independently reviewed by two neurointerventionists, and the diagnoses based on TOF MRA, S-n3DRA, and DSA were compared. The diagnostic efficacy (interobserver agreement and diagnostic performance) of S-n3DRA was compared with that of TOF MRA.

Results : S-n3DRA showed higher interobserver agreement $(\kappa=0.923)$ than TOF MRA $(\kappa=0.465)$ and significantly higher accuracy than MRA in distinguishing an aneurysm from an infundibulum ( $p=0.0039)$.

Conclusion : Compared to MRA, S-n3DRA could provide better screening accuracy and information for distinguishing an aneurysm from an infundibulum. Therefore, S-n3DRA has the potential to reduce the need for DSA.

Key Words : Source image based new three-dimensional rotational angiogram - Time-of-flight magnetic resonance angiography . Digital subtraction angiography $\cdot$ Intracranial aneurysm $\cdot$ Infundibulum.

\section{INTRODUCTION}

Subarachnoid hemorrhage (SAH) caused by a ruptured ce- rebral aneurysm results in a serious disability ${ }^{2,3,12)}$. To improve the clinical outcome, early diagnosis and intervention are imperative. The primary screening tests for detecting cerebral

\footnotetext{
- Received : December 2, 2020 •Revised : February 15, $2021 ・$ Accepted : March 10, 2021

- Address for reprints : Kwang-Chun Cho

Department of Neurosurgery, International St. Mary's Hospital, College of Medicine, Catholic Kwandong University, 25 Simgok-ro 100beon-gil, Seo-gu, Incheon 22711 , Korea Tel : +82-32-290-2988, Fax : +82-32-2903879, E-mail : ulyanminz@naver.com, ORCID : https://orcid.org/0000-0002-0261-9283
}

*H Jang and WS Jung contributed equally to this work as co-first authors.

This is an Open Access article distributed under the terms of the Creative Commons Attribution Non-Commercial License (http://creativecommons.org/licenses/by-nc/4.0) which permits unrestricted non-commercial use, distribution, and reproduction in any medium, provided the original work is properly cited. 
aneurysms in many neurovascular centers are computed tomographic angiography (CTA) and magnetic resonance angiography (MRA) ${ }^{7,9)}$. However, these screening techniques have limitations ${ }^{6,10,14)}$. Therefore, digital subtraction angiography (DSA) is considered the gold standard for a confirmatory diagnosis $^{13)}$. However, despite its accuracy, DSAs performed for suspected aneurysms, may misdiagnose an infundibulum as an aneurysm; expose patients to radiation from contrast me$\mathrm{dia}^{4,5,15)}$; and subject patients to an unnecessarily invasive procedure. A non-invasive diagnostic procedure with sufficient accuracy and low radiation exposure would therefore be ideal and may replace DSA as the diagnostic gold standard. In this study, we propose a new method that increases the rate of aneurysm detection and can distinguish an aneurysm from an infundibulum.

\section{MATERIALS AND METHODS}

\section{Data acquisition}

The study protocol was approved by Institutional Review Board of the Catholic Kwandong University International St. Mary's Hospital (IRB No. IS20RIMI0030). Patient informed consent was waived. We retrospectively reviewed the hospital and radiological records of consecutive patients who underwent DSA to confirm an antecedent MRA diagnosis of an unruptured aneurysm. Then, in this pilot study, we included only those patients who were diagnosed with an infundibulum and not aneurysm using the DSA. In total, 22 patients were included comprising 11 men and 11 women, ranging in age from 29 to 79 years (mean 57.3 years).

\section{On time-of-flight (TOF) MRA three-dimensional (3D) reconstruction images}

All 22 patients underwent MRAs as screening tests and were diagnosed as having aneurysms by the radiologists who reviewed the MRA results. Among these 22 patients, 23 lesions were evaluated by two interventionists. All MRA studies included the TOF source images. These TOF source images and $3 \mathrm{D}$ reconstructed MRA images were all used to diagnose aneurysms.

\section{Source image based new 3D rotational angiogra- phy (S-n3DRA) : image reconstruction}

We reconstructed new images using the DSA workstation. To reconstruct the new images, we used the TOF source images from the MRA. The thickness of these MRA source images was about $1 \mathrm{~mm}$. Approximately 150-160 source images were transported to a workstation (DELL PRECISION T3600, Allura 3D-RA 6.4.0 S/W; Philips Medical Systems, Eindhoven, Netherlands) connected to the DSA machine in the DICOM format. Although we used the Allura 3D-RA software in this study, it has been confirmed that S-n3DRA images could be reconstructed regardless of the type of software used in DSA workstation. We coined the term "source image based new three-dimensional rotational angiograms" for the newly reconstructed images used in this study, which were all reconstructed in approximately one minute.

\section{$3 \mathrm{D}$ rotational angiogram (3DRA) images acquisi- tion}

The 3DRA based on DSA, the gold standard for diagnosing cerebrovascular lesions, was used as a standard of reference. 3DRA images were obtained using biplane $\mathrm{C}$-arm digital angiography equipment (Allura Clarity FD 20/20; Philips Medical Systems) with a 20-inch field of view, a frame rate of $30 \mathrm{f} / \mathrm{s}$, and a rotation range of $270^{\circ}$.

The contrast medium (Visipaque-320; GE Healthcare, Cork, Ireland) was injected at a flow rate of $4 \mathrm{~mL} / \mathrm{s}$ for a total of $7 \mathrm{~mL}$ via a $5 \mathrm{~F}$ catheter positioned at the cervical portion of the internal carotid artery and/or vertebral artery for DSA imaging. For 3D DSA imaging, contrast medium was injected at a flow rate of $3 \mathrm{~mL} / \mathrm{s}$ for a total of $18 \mathrm{~mL}$ followed by a scan delay of 2 seconds.

The rotational angiography was performed with $270^{\circ}$ rotation of the C-arm in 4.1 seconds. Imaging data were automatically exported to the work station, where reconstruction of 3DRA was performed with a matrix of $384^{3}$ pixels.

3DRAs were produced by the workstation (DELL PRECISION T3600, Allura 3D-RA 6.4.0 S/W; Philips Medical Systems) using data from rotational angiographies.

\section{Comparison of Aneurysm and Infundibulum}

All images were interpreted by two neurointerventionists (J.J.K. and C.K.J.), who were blinded to the study. We compared the existing MRA images with the new images (S- 
n3DRA images) created from them using 3DRA as the reference. The presence of aneurysms or infundibulums on either the MRA (TOF source and 3D reconstructed MRA), Sn3DRA, or 3DRA images was assessed using a previously described three-point scale, and any interobserver disagreement was resolved by consensus. This scale reflects the confidence with which observers could distinguish aneurysms from infundibulums : 1) easy to judge, 2) difficult to judge, and 3) unable to judge. The interpretation of the images from MRA, Sn3DRA, and 3DRA was conducted at intervals of 3 months so as to allow enough time for the observers to forget the images.

Agreement between independent observers in each modality was determined by calculating $\kappa$ values (poor agreement, $\kappa=0$; slight agreement, $\kappa=0.01-0.20$; fair agreement, $\kappa=0.21-$ 0.40 ; moderate agreement, $\kappa=0.41-0.60$; good agreement, $\kappa=0.61-0.80$; excellent agreement, $\kappa=0.81-1.00$ ).

\section{Statistical analysis}

Fisher's exact probability test using MedCalc Statistical Software version 19.4.1 (MedCalc Software Ltd., Ostend, Bel- gium) was used to compare the ability of the different images in distinguishing aneurysms from infundibulums. $p<0.05$ was considered statistically significant.

\section{RESULTS}

S-n3DRA showed higher interobserver agreement $(\kappa=0.923)$ than MRA ( $\kappa=0.465)$. The Fisher's exact probability showed that S-n3DRA was significantly better than MRA at distinguishing aneurysms from infundibulums $(p=0.0039)$ (Table 1). The sensitivity of S-n3DRA (73.9\%) was higher than that of MRA (35.3\%).

\section{Case presentation}

\section{Case 1}

A 74-year-old woman was admitted to our hospital for the treatment of an unruptured aneurysm that was incidentally found on MRA. On TOF MRA 3D reconstruction images, a

Table 1. Comparison of MRA and S-n3DRA for differentiation between aneurysm and infundibulum

\begin{tabular}{|c|c|c|c|c|c|c|}
\hline \multirow{2}{*}{ Procedure } & \multicolumn{3}{|c|}{ Scale of difficulty for judgement } & \multirow{2}{*}{ All cases } & \multirow{2}{*}{$x^{2}$} & \multirow{2}{*}{$p$-value } \\
\hline & 1 & 2 & 3 & & & \\
\hline MRA & 6 & 15 & 2 & 23 & & \\
\hline S-n3DRA & 17 & 6 & 0 & 23 & 11.118 & 0.003 \\
\hline
\end{tabular}

MRA : magnetic resonance angiography, S-n3DRA : source image based new three-dimensional rotational angiography
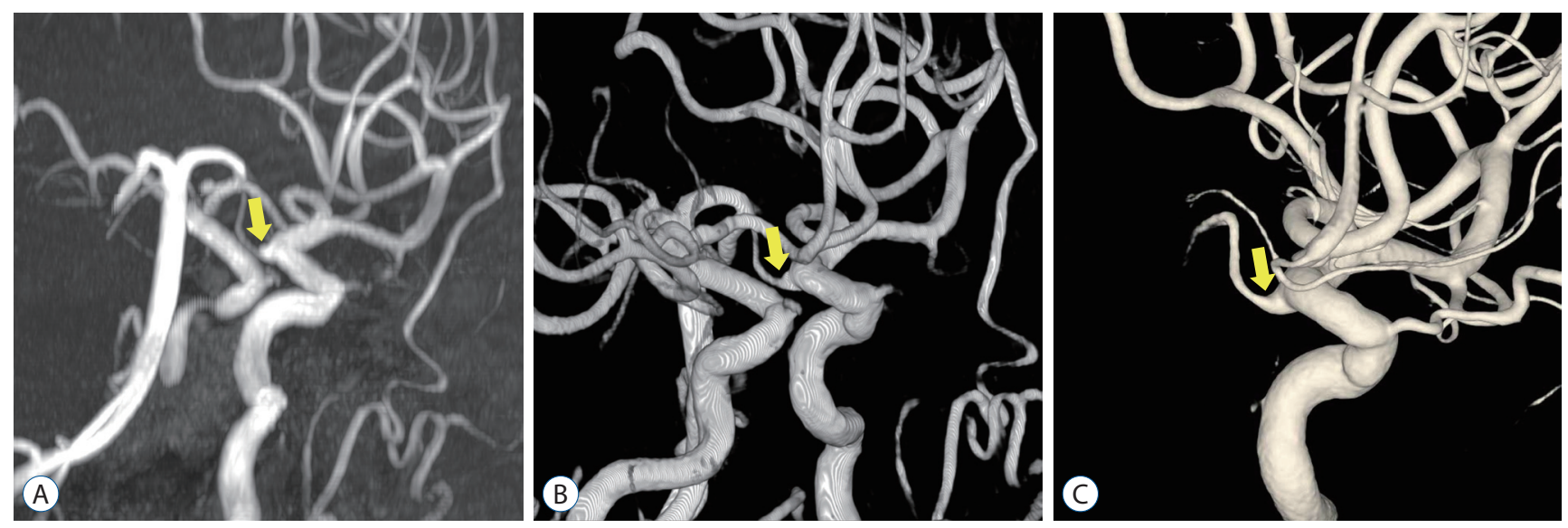

Fig. 1. A 74-year-old woman was admitted for the treatment of an unruptured aneurysm. A : On time-of-flight magnetic resonance angiography threedimensional (3D) reconstruction images, a saccular dilatation, possibly an aneurysm (arrow), was suspected near the posterior communicating artery (PcomA). B : Source image based new 3D rotational angiography showed that the PcomA originated from the wedge-shaped infundibulum (arrow). C : This lesion was confirmed as an infundibulum (arrow) on 3D rotational angiogram. 
saccular dilatation, with the possibility of being an aneurysm, was identified in the left internal carotid artery, and a nearby posterior communicating artery (PcomA) was also seen. However, its origin could not be clearly identified (Fig. 1A). Sn3DRA showed that the PcomA originated from the wedgeshaped infundibulum (Fig. 1B). This lesion was confirmed as an infundibulum on 3DRA of DSA (Fig. 1C).

\section{Case 2}

A 62-year-old man underwent an MRA for a medical checkup. On TOF MRA 3D reconstruction images, a saccular dilatation was identified in the anterior communicating artery
(Fig. 2A). However, S-n3DRA showed that the median artery of corpus callosum originated from the wedge-shaped infundibulum (Fig. 2B). This lesion was confirmed as an infundibulum on 3DRA of DSA (Fig. 2C).

\section{Case 3}

A 56-year-old woman presenting with a headache was examined using MRA. On TOF MRA 3D reconstruction images, a bulbous dilatation was identified in the right middle cerebral artery (MCA). A nearby branch was seen, but its origin could not be clearly discriminated (Fig. 3A). However, Sn3DRA showed that the MCA branch originated from the in-
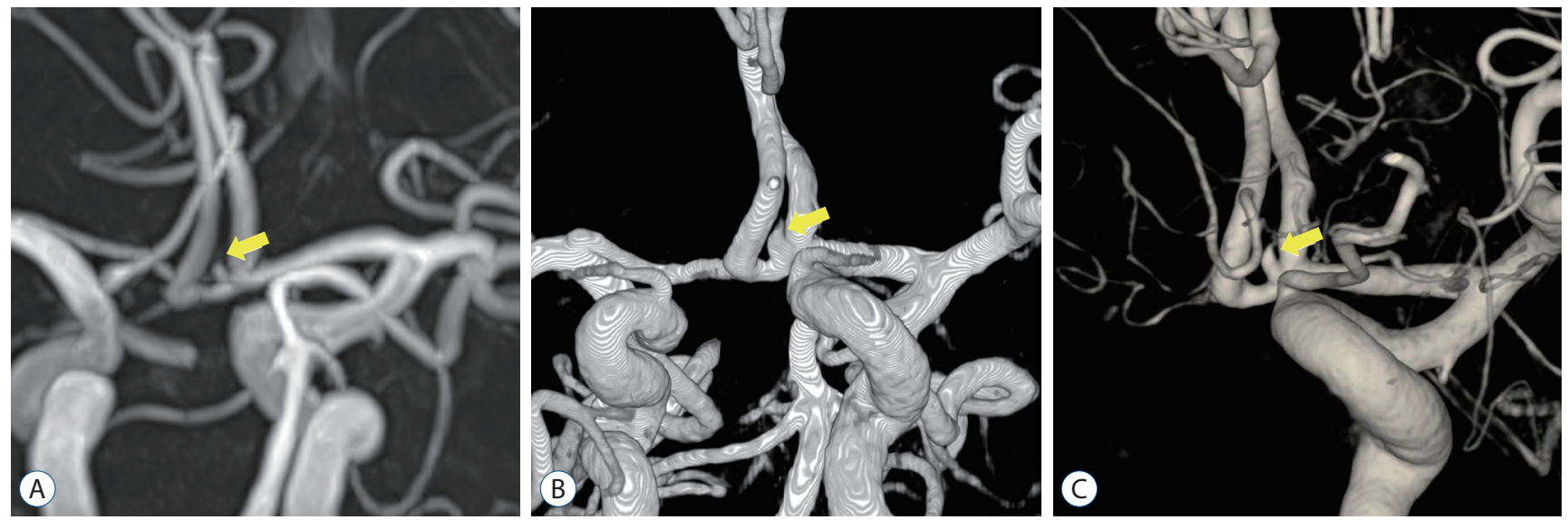

Fig. 2. A 62-year-old man underwent magnetic resonance angiography (MRA) for a medical check-up. A : On time-of-flight MRA three-dimensional (3D) reconstruction images, a saccular dilatation (arrow) was identified in the anterior communicating artery (AcomA). B : Source image based new 3D rotational angiography showed that the median artery of the corpus callosum originated from the wedge-shaped infundibulum (arrow). $\mathrm{C}:$ This lesion was confirmed as an infundibulum (arrow) on 3D rotational angiogram.
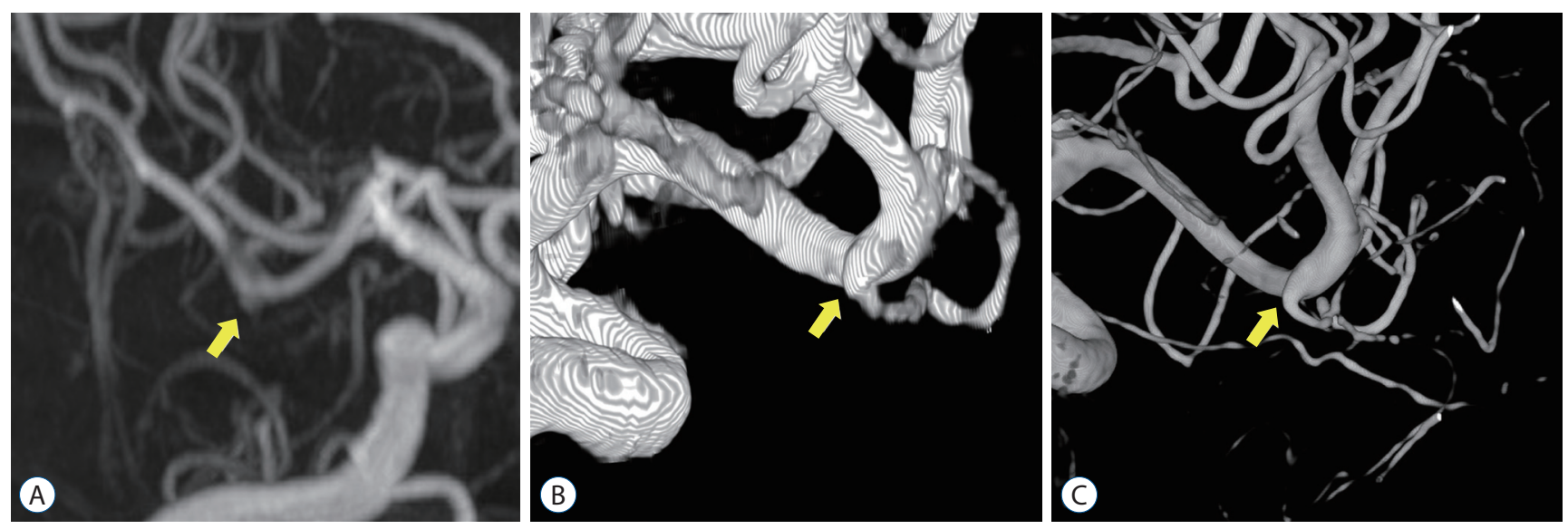

Fig. 3. A 56-year-old woman underwent magnetic resonance angiography (MRA) for a headache. A : On time-of-flight MRA three-dimensional (3D) reconstruction images, a bulbous dilatation (arrow) was identified in the right middle cerebral artery (MCA). B : Source image based new 3D rotational angiography showed that the MCA branch originated from the infundibulum (arrow). C: This lesion was confirmed as an infundibulum (arrow) on 3D rotational angiogram. 
fundibulum (Fig. 3B). This lesion was confirmed as an infundibulum on 3DRA of DSA (Fig. 3C).

\section{DISCUSSION}

In order to avoid the poor prognosis associated with ruptured aneurysms, unruptured aneurysms should be treated selectively before they rupture. Furthermore, these unruptured aneurysms should be diagnosed accurately by distinguishing them from infundibulums. Diagnostic tests with high accuracy, such as DSA, are preferable. The possibility of side-effects, however, caused by contrast medium and radiation ${ }^{4,5,15)}$, precludes DSA from being conducted indiscriminately.

MRA is the most commonly used screening test, but it is limited in its capability to distinguish aneurysms from infundibulums ${ }^{1,8,11}$. In fact, a significant number of patients visit higher-level hospitals for more accurate DSA testing following an MRA scan. However, suspected aneurysms are often found to be non-aneurysms on the DSA ${ }^{10}$. An accurate, non-invasive and safer means of screening would obviate the need for DSA, thereby preventing its side-effects.

\section{Complications of DSA}

DSA is an invasive test with potentially severe complications ${ }^{1,5)}$. In addition, there are side-effects from radiation exposure or use of contrast media ${ }^{5}$. The most common complication is groin hematoma ( $4.2 \%$ of examinations); systemic complications such as nausea, vomiting, and transient hypotension and anaphylaxis and death also occur ${ }^{5}$. Neurologic complications include transient ischemia attack (2.9\%), reversible ischemic neurologic deficit $(0.36 \%)$, and permanent stroke $(0.14 \%)^{5}$.

Although the frequency of these complications is decreasing gradually, their risk is still significant. Therefore, it is necessary to reduce the utilization of DSA whenever possible.

\section{Accuracy of S-n3DRA}

Results from our study suggests that S-n3DRA may be more superior as a screening test than MRA. Rather, the S-n3DRA is more similar to 3DRA, the gold standard, and thus can be valuable as a screening test (Table 1). S-n3DRA demonstrated excellent interobserver agreement $(\kappa=0.923)$ and significantly better ability than MRA in distinguish the aneurysms from infundibulums.

\section{Strength of S-n3DRA}

S-n3DRA retains the benefits of 3DRA. As in 3DRA, we can freely rotate S-n3DRA at various angles through post-processing techniques, thereby providing an arbitrary viewing angle. In addition, obscuring surrounding structures can be removed in volume rendered images, resulting in excellent visualization of the relationship between arteries and an aneurysm.

Based on the sensitivity of MRA (35.3\%) and S-n3DRA (73.9\%), the use of S-n3DRA would reduce additional DSA. Because S-n3DRA obviates the need for confirmatory DSA imaging, patients are spared from possible complications stemming from radiation exposure and arterial catheterization.

Using S-n3DRA does not require patients to undergo further scans and only requires the reconstitution of MRA images using a DSA workstation program. S-n3DRA therefore is potentially less time consuming and less financially burdensome.

\section{Application of S-n3DRA}

S-n3DRA may be a suitable diagnostic tool for patients with relative contraindications to DSA - old age, poor health, or anatomic difficulties such as proximal vessel tortuosity or aortic graft stent insertion status. With future research resulting in enhanced S-n3DRA accuracy, 3DRA may be replaced in some cases. S-n3DRA may also be implemented to further evaluate aneurysms found on MRA for size, shape and anatomical location.

\section{Limitation of S-n3DRA}

Currently, S-n3DRA images can be constructed using only a Philips workstation. However, research is underway to enable use of other workstations. Although MRA images were used to create S-n3DRA in this study, CTA based S-n3DRA may also be possible.

This study is limited by its retrospective design and small sample size. In addition, our results may have been affected by inter- or intra-observer bias. However, despite these limitations, the results of this study are valuable, and S-n3DRA is expected to compensate for MRA in the differentiation of an- 
eurysms and infundibulum. Because this is a pilot study, further research is needed to validate the usefulness of S-n3DRA.

\section{CONCLUSION}

S-n3DRA could provide excellent screening accuracy and better information in distinguishing aneurysms from infundibulum than MRA. This technique may reduce the use of DSA.

\section{CONFLICTS OF INTEREST}

No potential conflict of interest relevant to this article was reported.

\section{INFORMED CONSENT}

This type of study does not require informed consent.

\section{AUTHOR CONTRIBUTIONS}

\author{
Conceptualization : KCC \\ Data curation : JJK, CKJ, KCC \\ Formal analysis: WSJ \\ Methodology : SUM \\ Project administration : KCC \\ Visualization: WSJ \\ Writing - original draft : HJ, WSJ \\ Writing - review \& editing: HJ, WSJ, KCC
}

\section{ORCID}

Hyeongyu Jang https://orcid.org/0000-0001-5766-4383

Woo Sang Jung https://orcid.org/0000-0002-0727-3744

Seong Uk Myoung https://orcid.org/0000-0002-9328-1723

Jung-Jae Kim https://orcid.org/0000-0002-4669-8577

Chang Ki Jang https://orcid.org/0000-0001-8715-8844

Kwang-Chun Cho https://orcid.org/0000-0002-0261-9283

\section{References}

1. Adams WM, Laitt RD, Jackson A : The role of MR angiography in the pretreatment assessment of intracranial aneurysms: a comparative study. AJNR Am J Neuroradiol 21 : 1618-1628, 2000

2. Bederson JB, Connolly ES Jr, Batjer HH, Dacey RG, Dion JE, Diringer MN, et al. : Guidelines for the management of aneurysmal subarachnoid hemorrhage: a statement for healthcare professionals from a special writing group of the Stroke Council, American Heart Association. Stroke 40 : 994-1025, 2009

3. Brisman JL, Song JK, Newell DW : Cerebral aneurysms. N Engl J Med 355 : 928-939, 2006

4. Fifi JT, Meyers PM, Lavine SD, Cox V, Silverberg L, Mangla S, et al. : Complications of modern diagnostic cerebral angiography in an academic medical center. J Vasc Interv Radiol 20 : 442-447, 2009

5. Kaufmann TJ, Huston J 3rd, Mandrekar JN, Schleck CD, Thielen KR, Kallmes DF : Complications of diagnostic cerebral angiography: evaluation of 19,826 consecutive patients. Radiology 243 : 812-819, 2007

6. Menke J, Larsen J, Kallenberg K : Diagnosing cerebral aneurysms by computed tomographic angiography: meta-analysis. Ann Neurol 69 : 646-654, 2011

7. Numminen J, Tarkiainen A, Niemelä M, Porras M, Hernesniemi J, Kangasniemi $M$ : Detection of unruptured cerebral artery aneurysms by MRA at 3.0 tesla: comparison with multislice helical computed tomographic angiography. Acta Radiol 52 : 670-674, 2011

8. Okahara M, Kiyosue H, Yamashita M, Nagatomi H, Hata H, Saginoya T, et al. : Diagnostic accuracy of magnetic resonance angiography for cerebral aneurysms in correlation with 3D-digital subtraction angiographic images: a study of 133 aneurysms. Stroke 33 : 1803-1808, 2002

9. Sailer AM, Wagemans BA, Nelemans PJ, de Graaf R, van Zwam WH : Diagnosing intracranial aneurysms with MR angiography: systematic review and meta-analysis. Stroke 45 : 119-126, 2014

10. Satoh T, Omi M, Ohsako C, Fujiwara K, Tsuno K, Sasahara W, et al. : Differential diagnosis of the infundibular dilation and aneurysm of internal carotid artery: assessment with fusion imaging of 3D MR cisternography/angiography. AJNR Am J Neuroradiol 27 : 306-312, 2006

11. Satoh T, Onoda K, Tsuchimoto $S$ : Visualization of intraaneurysmal flow patterns with transluminal flow images of 3D MR angiograms in conjunction with aneurysmal configurations. AJNR Am J Neuroradiol 24 : 1436-1445, 2003

12. van Gijn J, Kerr RS, Rinkel GJ : Subarachnoid haemorrhage. Lancet 369 : 306-318, 2007

13. van Rooij WJ, Sprengers ME, de Gast AN, Peluso JP, Sluzewski M : 3D rotational angiography: the new gold standard in the detection of additional intracranial aneurysms. AJNR Am J Neuroradiol 29 : 976-979, 2008

14. Wermer MJ, van Walderveen MA, Garpebring A, van Osch MJ, Versluis MJ : 7Tesla MRA for the differentiation between intracranial aneurysms and infundibula. Magn Reson Imaging 37 : 16-20, 2017

15. Willinsky RA, Taylor SM, TerBrugge K, Farb RI, Tomlinson G, Montanera $W$ : Neurologic complications of cerebral angiography: prospective analysis of 2,899 procedures and review of the literature. Radiology $227: 522-528,2003$ 